\title{
Symmetries of spacetime and their relation to initial value problems*
}

\author{
István Rácz \\ MTA KFKI, Részecske- és Magfizikai Kutatóintézet \\ H-1121 Budapest, Konkoly Thege Miklós út 29-33. \\ Hungary \\ E-mail: istvan@sunserv.kfki.hu
}

November 2, 2018

\begin{abstract}
We consider covariant metric theories of coupled gravity-matter systems satisfying the following two conditions: First, it is assumed that, by a hyperbolic reduction process, a system of first order symmetric hyperbolic partial differential equations can be deduced from the matter field equations. Second, gravity is supposed to be coupled to the matter fields by requiring that the Ricci tensor is a smooth function of the basic matter field variables and the metric. It is shown then that the "time" evolution of these type of gravity-matter systems preserves the symmetries of initial data specifications.
\end{abstract}

\section{Introduction}

In a recent paper [19] the necessary and sufficient conditions ensuring the existence of Killing vector fields in covariant metric theories of coupled gravity matter systems were investigated. In that work matter fields were assumed to satisfy quasi-linear wave equations and to possess minimal coupling to gravity. In addition, gravity was supposed to be coupled to the matter fields by requiring that the Ricci tensor is a function of the basic matter field variables, their first covariant derivatives and the metric. Within this framework it was demonstrated that symmetries of initial data specifications are preserved by "time" evolution.

The pragmatic aim in [19] was to justify some of the claims of [9, 18] concerning the existence of Killing vector fields in the characteristic initial problem in case of Einstein-Klein-Gordon, Einstein[non-Abelian] Higgs or Einstein-[Maxwell]-Yang-Mills-dilaton systems. The matter field variables] of these particular Einstein-matter systems all satisfy quasi-linear wave equations. However, the evolution of various matter fields, such as perfect- or dissipative fluids and spinor fields, is not governed by quasi-linear wave equations; rather they satisfy first order symmetric hyperbolic equations.

Clearly, it is of obvious physical interest to enlarge the framework of [19] to include matter fields satisfying equations of this more general type and to determine those conditions which guarantee the existence of a Killing vector field for the associated gravity-matter systems. This is, in fact, our principal aim in this paper which is organized as follows. The next section is to specify the

\footnotetext{
* This research was supported in part by OTKA grant T034337.

${ }^{1}$ In case of gauge fields they are yielded by suitable gauge fixing (see e.g. [19, 18 for further details).
} 
class of gravity-matter systems to which our main result applies. In particular, a representation of the physical fields and the relevant basic field equations are chosen so that well-posed initial value problem can be associated with the selected gravity-matter systems. Section 3 is to study all the equations relevant for the 'evolution' of the Lie derivatives, with respect to a 'candidate Killing vector field', of our basic variables. This section includes our main result justifying the claim that the "time" evolution of the selected gravity-matter systems preserves the symmetries of initial data specifications. Finally, section 1 contains our concluding remarks. In particular, issues associated with the evolution of matter fields on fixed geometrical backgrounds and the possible affection of a non-dynamical field on the evolution of symmetries are considered.

\section{The gravity-matter systems}

In order to have a suitable framework for the study of spacetime symmetries, first of all we give a mathematically precise specification of the gravity-matter theories to which our main result will apply. Obviously, it is impossible to recall details of all the particular systems which have ever been studied. Instead, a general, and thereby flexible, enough framework is chosen within which common features of a reasonably large set of particular theories can be investigated. The model specified below is highly influenced by the fundamental study of Geroch [11], where a universal treatment of physical fields and equations is presented.

\subsection{The choice of basic variables and field equations}

The physical fields are supposed to be represented by smooth tensor fields on a smooth fourdimensional paracompact orientable manifold $M$. In particular, the "gravitational field" is assumed to be represented by a smooth Lorentzian metric field $g_{a b}$ on $M$ and all the gravitational effects are expected to be described in terms of this metric and covariant quantities derivable from it. It is known that other basic variables such as tetrads or spin frames could also be used to represent gravity. However, since our eventual aim in this work is to study spacetime symmetries the use of $g_{a b}$ suits the most.2

In general, tensor fields representing the matter content of a theory will be denoted by $T_{(i)}^{\mathcal{A}}$, where $i$ in round brackets is a name index taking values from the set $\{1,2, \ldots, N\}$, and the capital script letter $\mathcal{A}$ stands for a composite (abstract) index. The constituents of $\mathcal{A}$, i.e. the exact form $T_{(i)}^{a_{1} \ldots a_{k_{i}}}{ }_{b_{1} \ldots b_{l_{i}}}$ of the $\left(k_{i}, l_{i}\right)$ type tensor field $T_{(i)}^{\mathcal{A}}$ will be spelled out explicitly in all the non-selfexplaining situations. The matter fields might also have gauge dependence but, even if they have, it will be assumed that suitable gauge choices have been made so that they can be considered as fields on the spacetime manifold. Thereby the relevant gauge or internal space indices will be suppressed.

The evolution of the fields $T_{(i)}^{\mathcal{A}}$ is supposed to be given in terms of partial differential equations. In particular, $T_{(i)}^{\mathcal{A}}$ are assumed to solve systems of first order quasi-linear partial differential equations of the form

$$
\sum_{(j)} K_{(i)(j)}^{\mathcal{A} e} \mathcal{B} \nabla_{e} T_{(j)}^{\mathcal{B}}+L_{(i)}^{\mathcal{A}}=0
$$

were $\nabla_{a}$ denotes the unique (torsion free) covariant derivative operator associated with the spacetime metric $g_{a b}$. Moreover, the coefficients $K_{(i)(j)}^{\mathcal{A}} \mathcal{B}$ and $L_{(i)}^{\mathcal{A}}$ in (2.1) are expected to be smooth fields on a tensor bundle, $\mathcal{T}$, built up from the fields $T_{(i)}^{\mathcal{A}}$ and $g_{a b}$.

\footnotetext{
${ }^{2}$ Unless otherwise stated we shall use the notation and conventions of [21].
} 
It is worth emphasizing that this framework covers matter systems like Klein-Gordon fields, Maxwell fields, perfect fluids and various other type of matter fields, along with their conventional couplings (see for more details Appendix A of [11|). In fact, it seems to cover virtually every partial differential equation in physics. f $^{3}$ In particular, quasi-linear wave equations of the form

$$
\nabla^{f} \nabla_{f} T_{(i)}^{\mathcal{B}}+F_{(i)}^{\mathcal{B}}\left(g_{c d}, T_{(j)}^{\mathcal{C}}, \nabla_{c} T_{(j)}^{\mathcal{C}}\right)=0,
$$

can immediately be put into the form of (2.1), by following a standard procedure (see e.g. pages 76-77 of [10]) which starts, by adding the first derivatives $T_{(N+i)}^{\mathcal{A}} e=\nabla_{e} T_{(i)}^{\mathcal{A}}$ to the basic variables.

Note also that, as indicated above, in case of matter fields, like Yang-Mills or Higgs fields with a gauge freedom, to get the desired framework a gauge fixing is required to be made. For instance, in case of a Yang-Mills field the use of the generalized Lorentz gauge condition is needed to put the Yang-Mills equations into the form of a quasi-linear wave equation (see for details e.g. [7, 19]), which can then be recast into the form of (2.1) by the above mentioned procedure.

According to the above assumptions matter fields are assumed to be coupled to gravity through the dependence of $K_{(i)(j)}^{\mathcal{A} e} \mathcal{B}$ and $L_{(i)}^{\mathcal{A}}$ on the metric, as well as, via the presence of the metric compatible covariant derivative operator $\nabla_{e}$ in (2.1). Concerning the coupling of the geometry to matter fields, we shall require that the Ricci tensor $R_{a b}$ be a smooth function of the matter field variables and the metric,

$$
R_{a b}=R_{a b}\left(T_{(i)}^{\mathcal{A}}, g_{m n}\right) .
$$

Note that this assumption immediately imposes a restriction, in addition to (2.1), on the fields $T_{(i)}^{\mathcal{A}}$ via the twice contracted Bianchi identity.

It is straightforward to see that the conditions so far have been made are satisfied by all the 'customary' Einstein-matter systems. In particular, the present framework immediately covers the setting applied in 19 since the matter field variables used here includes the first covariant derivatives of the basic matter field variables applied there. Moreover, the selected model is general enough to host e.g. the 'conformally equivalent representation' of higher curvature theories associated with a gravitational Lagrangian that is a polynomial of the Ricci scalar (for further details see e.g. [12]).

In addition to the above assumptions we shall impose the following requirement which plays a significant role in the derivation of our main result and which is usually made implicitly in most of the related discussions.

Condition 2.1 The fields $K_{(i)(j)}^{\mathcal{A} \text { B }}$ and $L_{(i)}^{\mathcal{A}}$, along with the Ricci tensor when it is viewed as a functional of the basic variables as the expression appearing on the r.h.s. of (2.3), are assumed to be smooth functionals on the tensor bundle $\mathcal{T}$ depending exclusively on $T_{(i)}^{\mathcal{A}}$ and $g_{a b}$,

$$
K_{(i)(j)}^{\mathcal{A} e} \mathcal{B}=K_{(i)(j)}^{\mathcal{A} e} \mathcal{B}\left(T_{(k)}^{\mathcal{C}}, g_{m n}\right) \quad \text { and } \quad L_{(i)}^{\mathcal{A}}=L_{(i)}^{\mathcal{A}}\left(T_{(j)}^{\mathcal{C}}, g_{m n}\right)
$$

without having any explicit dependence on the points of $M$.

\footnotetext{
${ }^{3}$ In principal, spinor fields could also be present among the allowed matter field variables. This is particularly so, because the Dirac equations possess the form of (2.1). Moreover, it was demonstrated by Friedrich and Rendall 10 that symmetric hyperbolic equations can be built up from the Einstein-Dirac system. The main purpose of this work, however, is to study the existence of symmetries which requires the use of Lie derivatives of all the fields with respect to essentially arbitrary vector fields on $M$. On the other hand, it is known that the Lie derivative of spinor fields cannot be defined (at least not in the conventional way) with respect to arbitrary vector fields but only to conformal Killing vector fields. Before the procedure described below can be extended to Einstein-Dirac systems as well one should clear up e.g. how to make a sensible variation of a composite metric-spinor system. Obviously, there is no problem of varying the spinor field while the metric is kept fixed. However, a procedure of complete generality, such as 'changing the metric while keeping the spinor field fixed' does not exist.
} 
This assumption expresses the idea that the distinction between two points of a spacetime manifold $M$ is rooted in the difference between the values of some physical field at that points. In other words, the points of the spacetime $\left(M, g_{a b}\right)$ are assumed to be identified by observing some physical fields not by somehow "perceiving the points of $M$ themselves directly" |11|. Notice, however, that whenever the dynamics of certain fields $T_{(i)}^{\mathcal{A}}$ on a fixed background spacetime is considered the coefficients $K_{(i)(j)}^{\mathcal{A} e} \mathcal{B}$ and $L_{(i)}^{\mathcal{A}}$ will immediately pick up an 'explicit' spacetime dependence through that of $g_{a b}$.

Note finally that the relations (2.1) and (2.3), along with condition 2.1, manifest an important property of the composite gravity-matter system. Namely, they guarantee that the coupling of all the subsystems rests upon only zero order terms of the relevant basic variables.

\subsection{Hyperbolic reduction of the field equations}

A key feature of the field equations of physics is that a system of partial differential equations can be deduced from them so that, for suitable initial data specifications, this equations yield the 'time' evolution of the relevant system. This subsection is to impose further conditions which guarantee the well-posed initial value problems can be associated with the selected gravity-matter theories.

\subsubsection{Symmetric hyperbolic systems for the matter fields}

To attain 'existence and uniqueness results' relevant for an initial value problem associated with the gravity-matter systems of the above type first the basic field equations have to be put into a specific form of PDEs for which existence and uniqueness of solutions is guaranteed. In this work first order symmetric hyperbolic PDEs are used for this purpose which are specified as follows [10]:

Consider, as our unknown, a function $\Phi$ defined on an open subset of $\mathbb{R}^{4}$ and assume that it takes values in a finite dimensional vector space $V$. The hyperbolic systems we are interested in now are of the form

$$
A^{\gamma} \partial_{\gamma} \Phi+B=0
$$

where $A^{\gamma}=A^{\gamma}(x, \Phi)$ are supposed to be smooth functions defined on an open subset of $\mathbb{R}^{4} \times V$ taking values in the vector space $L(V)$ of linear maps of $V$ to itself, while $B=B(x, \Phi)$ is expected to be a smooth function on an open subset of $\mathbb{R}^{4} \times V$ with values in $V$. The basic requirement on the system to be called symmetric hyperbolic is that the $A^{\gamma}$ are symmetric, with respect to some inner product on $V$, and there exists a covector field $\xi_{\gamma}$ such that $A^{\gamma} \xi_{\gamma}$ is positive definite for all admissible values of $\Phi$.

By imposing the following condition we shall restrict our considerations to matter fields for which a symmetric hyperbolic system can be deduced from (2.1).

Condition 2.2 Equation (2.1) can be split into a symmetric hyperbolic system of the form (2.5), in terms of a suitable variable $\Phi$ labeling cross-sections of the tensor bundle built up from the fields $T_{(i)}^{\mathcal{A}}$, and constraint equations which, along with the possible gauge conditions, propagate under the evolution of the associated hyperbolic system.

Note that there is no known a general practical procedure which would immediately produce a symmetric hyperbolic system of the form (2.5) from (2.1). In fact, the actual way of deducing a symmetric hyperbolic system (2.5) from (2.1) and the justification that the constraints also propagate for each particular case might be very complicated and most of the time requires very special 
considerations. However, the actual way of getting the required hyperbolic reduction of the selected matter system will not play any role in the basic argument concerning the evolution of symmetries of initial data specifications.

Notice also that even after imposing condition 2.2 the framework we have still covers matter systems like Klein-Gordon, dilaton, Higgs, Maxwell-Yang-Mills fields fields and various fluids along with the conventional couplings of these systems. In fact, this setting is suitable to host virtually all the physically relevant matter systems for which a well-posed initial value problem can ever be formulated.

\subsubsection{Hyperbolization of the gravitational part}

To get symmetric hyperbolic equations relevant for the selected gravity-matter system we need to take care beside the hyperbolic reduction of (2.1) that of (2.3), as well. It is known [11, 10] that, due to the general covariance of the considered metric theories of gravity, there is no way to get hyperbolic evolution from the coupled field equations (2.1) and (2.3) without fixing the gauge associated with the diffeomorphism invariance. One of the possibilities to make such a gauge fixing rests on the form of the Ricci tensor $R_{a b}$ given in local coordinates $x^{\alpha}$ as

$$
R_{\alpha \beta}=-\frac{1}{2} g^{\mu \nu} \partial_{\mu} \partial_{\nu} g_{\alpha \beta}+g_{\delta(\alpha} \partial_{\beta)} \Gamma^{\delta}+H_{\alpha \beta}\left(g_{\varepsilon \rho}, \partial_{\gamma} g_{\varepsilon \rho}\right),
$$

and it is done by simply replacing $\Gamma^{\delta}=g^{\gamma \rho} \Gamma_{\gamma \rho}^{\delta}$ in (2.6) with arbitrary 'gauge source functions' $f^{\delta}$ (chosen so that $f^{\delta}=\Gamma^{\delta}$ is satisfied on initial hypersurfaces). The equations yielded by substituting, in local coordinates, the r.h.s. of (2.6) for the l.h.s of (2.3), with $\Gamma^{\delta}$ replaced by $f^{\delta}$, are refereed to as reduced gravity equations. These equations can be put into the form of a first order symmetric hyperbolic equation by introducing all first order derivatives of $g_{\alpha \beta}$ as new variables and let $\Phi$ consists of $g_{\alpha \beta}$ together with these derivatives. Then by making use of the reduced gravity equations a system of symmetric hyperbolic equations for $\Phi$ can be built up by a standard procedure (see for more details e.g. $|4,10|)$.

\subsubsection{Hyperbolic reduction of the coupled system}

Upon having a hyperbolic reduction of the matter and gravity equations separately, it is important to know whether the coupled equations also form a symmetric hyperbolic system. The answer is in the affirmative. This can be justified by recalling that the coupling of the matter field equations (2.1) - which by condition 2.2 can be cast into a symmetric hyperbolic system of the form (2.5) and that of a fixed hyperbolic reduction of 2.3 - written in first order symmetric hyperbolic form is only by terms of order zero, hence, the combined system is also symmetric hyperbolic. Therefore, local existence and uniqueness results immediately apply to the relevant reduced gravity-matter equations in case of regular initial value problems.

There is only one significant requirement the applied initial value problem has to satisfy: The existence and uniqueness of solutions to symmetric hyperbolic equations is expected to be guaranteed within its framework in the smooth setting. The initial value problems which satisfy this condition are referred to as regular initial value problems. Immediate examples for appropriate initial value problems are the standard Cauchy problem (see e.g. Ref. [4]) and also the characteristic initial value problem associated with an initial hypersurface represented by either the union of two smooth null hypersurfaces intersecting on a 2-dimensional spacelike surface [17, 20] or a characteristic cone

\footnotetext{
${ }^{4}$ For an excellent review of all the related issues see $[8]$.
} 
[6, 1, 20]. Since no further requirement on the initial value problem is used anywhere in the derivation of our results we shall not make a definite choice among these regular initial value problems. Thereby the following notation will be applied on equal footing to any of these initial value problems: The initial hypersurface will be denoted by $\Sigma$, while the initial data on $\Sigma$ will be represented by the basic variables in square brackets. In particular, an initial data specification for an equation of the form (2.5) is represented by the pair $(\Sigma,[\Phi])$.

As mentioned above the hyperbolic reduction is done by splitting the basic equations to pure evolution equations and constraint ones. In fact, the constraint equations, along with the gauge conditions, manifest certain (differential) identities that have to be satisfied by $T_{(i)}^{\mathcal{A}}$ and $g_{a b}$. In particular, they impose restrictions on the possible initial data sets. Thereby, having a hyperbolic reduction of the coupled system suitable initial data consistent with the relevant gauge conditions and satisfying all the constraints has to be arranged. Finally, it has also to be demonstrated that the gauge conditions and the constraints propagate with the 'time' evolution of the relevant coupled reduced system. In the present case, by making use of a suitable combination of condition 2.2 and the twice contracted Bianchi identity (see also page 20 and pages 76-77 of [10]), it can be justified that the gauge conditions and the constraints will be satisfied throughout the domain of dependence associated with a solution to the coupled evolution equations if they hold on the initial data surface.

\section{The evolution of symmetric initial data specifications}

Consider a gravity-matter system of the type specified in the previous section satisfying conditions 2.1 and 2.2. Assume that a regular initial value problem has been chosen, moreover, a suitable initial data set relevant for the coupled symmetric hyperbolic evolution equations also satisfying the pertinent gauge conditions and constraint equations has also been selected. Recall that then we immediately have a detailed knowledge of the metric and matter fields on the initial data surface $\Sigma$ since in any of the regular initial value problems $T_{(i)}^{\mathcal{A}}$ and $g_{a b}$, along with their derivatives up to arbitrary order, can always be determined on $\Sigma$ by making use of an initial data specification, $\left(\Sigma,\left[T_{i i}^{\mathcal{A}}\right],\left[g_{a b}\right]\right)$, and the field equations. Clearly, it is of obvious interest to identify those initial data sets that are guaranteed to evolve to configurations possessing certain type of symmetries. The rest of this paper is to make it clear under what conditions the symmetries, and more importantly what sort of symmetries, of initial data sets will be inherited by the corresponding solutions.

There are various possibilities which make it sensible to consider an initial data specification to be symmetric. The most straightforward one is the following: An initial data set $\left(\Sigma,\left[T_{(i)}^{\mathcal{A}}\right],\left[g_{a b}\right]\right)$ is considered to be symmetric if there exists a diffeomorphism $\psi: \Sigma \rightarrow \Sigma$ of the initial hypersurface $\Sigma$ onto itself so that it leaves the initial data invariant. These type of symmetries of initial data specifications were considered in $[10$, for the case of Einstein-vacuum systems. Based essentially on the uniqueness of maximal Cauchy developments, it was demonstrated there that such a symmetry of a vacuum initial data set will be inherited by the corresponding solution. By making use of a straightforward adaptation of this argument (see, for more details, pages 90-91 of [10]) to the case of gravity-matter systems investigated here, it can also be justified that a symmetry, of the considered type, of an initial data set gives also rise to a symmetry of the associated Cauchy development, i.e. there will exist a diffeomorphism $\Psi: M \rightarrow M$ with $\Psi_{*} T_{(i)}^{\mathcal{A}}=T_{(i)}^{\mathcal{A}}$ and $\Psi_{*} g_{a b}=g_{a b}$ with $\left.\Psi\right|_{\Sigma}=\psi$.

Since there are symmetries of Cauchy developments which does not necessarily map any initial data surfaces onto themselves it is of obvious interest to know whether the traces of these more general type of symmetries can also be recognized by making use of initial data specifications. It seems to be hopeless to find an evidence for the existence of these more general type of symmetries in the case of discrete symmetry transformations. However, whenever a gravity-matter system admits 
a Killing vector field, i.e. there exists, at least locally, a one-parameter family of isometry actions in a neighbourhood of $\Sigma$, the procedure described in detail in the following section can be applied.

Before approaching to this point there are two comments in order. Firstly, I would like to emphasize the complementary character of the procedures associated with the above mentioned two types of symmetries. The results relevant for the more general type of symmetries obviously cover the special case whenever an initial data specification admits a one-parameter family of diffeomorphisms $\psi_{\tau}: \Sigma \rightarrow \Sigma$ leaving the initial data invariant. However, it has its drawback not being able to handle the case of discrete isometry actions which, on the other hand, can be covered by the indicated generalization of the method of Friedrich and Rendall [10] in the particular case when the initial data surface is mapped onto itself by the associated diffeomorphism.

Secondly, the results presented below are due to straightforward adaptations of earlier methods applied in the Einstein-vacuum case to study the linearization stability problem by Moncrief [14, 15] (see also [3, 21]). Note that these results provide an immediate generalization of the main assertion of [19], and motivates a possible further strengthening of the conclusion of [13], since the results covered by [19, 13] are relevant merely for gravity-matter systems with matter fields satisfying quasi-linear wave equations.

\subsection{The construction of a candidate Killing vector field}

It is known that if we had a Killing vector field $K^{a}$, satisfying the Killing equation,

$$
\mathcal{L}_{K} g_{a b}=\nabla_{a} K_{b}+\nabla_{b} K_{a}=0
$$

and the integrability condition,

$$
\nabla_{a} \nabla_{b} K_{c}+R_{b c a}^{d} K_{d}=0
$$

of the 2-form field $\Xi_{a b}=\nabla_{a} K_{b}$ this Killing vector field would be completely determined by the values of $K_{a}$ and $\nabla_{a} K_{b}$ at any point of $M$ since the above two equations imply a system of ODE's for the components of $K_{a}$ and $\nabla_{a} K_{b}$ along any $C^{1}$ curve. However, the existence of a Killing vector field cannot be proven in this way. Nevertheless, the contraction,

$$
\nabla^{e} \nabla_{e} K^{a}+R_{d}^{a} K^{d}=0,
$$

of (3.2), which is a linear homogeneous wave equation for $K^{a}$, provides the means to construct a 'candidate' Killing vector field. Obviously, any Killing vector field satisfies (3.3) but not all of its solutions will give rise to a Killing vector field.

\subsection{Evolution of the Lie derivatives of the basic variables}

The conditions ensuring the existence of initial data $\left[K^{a}\right]$ on an initial hypersurface $\Sigma$ so that the unique solution $K^{a}$ of (3.3) will be a Killing vector field are necessarily given in terms of restrictions on the values of the fields $T_{(i)}^{\mathcal{A}}$ and $g_{a b}$ on $\Sigma$. The relevant requirements can be read off the evolution equations for the Lie derivatives of our basic variables. The remaining part of this section is to identify these conditions and thereby we shall prove the following:

Theorem: Let $\left(M, g_{a b}\right)$ be a spacetime associated with a gravity-matter system as it was specified in section $\mathbf{G}$. Denote by $D[\Sigma]$ the domain of dependence of an initial hypersurface $\Sigma$ associated with a regular initial value problem. Then there exists a non-trivial Killing vector field $K^{a}$ on $D[\Sigma]$, so that the matter fields are also invariant, i.e. $\mathcal{L}_{K} T_{(i)}^{\mathcal{A}}=0$, if there exists a non-trivial initial data set $\left[K^{a}\right]$ for 3.35 so that $\mathcal{L}_{K} T_{(i)}^{\mathcal{A}}, \mathcal{L}_{K} g_{a b}$ and $\nabla_{c}\left(\mathcal{L}_{K} g_{a b}\right)$ all vanish identically on $\Sigma$. 
Before presenting the proof of this theorem let us emphasize certain points relevant for the applied setting. First of all, the practical problem of finding initial data $\left[K^{a}\right]$ for $(3.3)$ - which in each particular case is the hard part of the work - is not considered here. It is, however, important to note that, upon having any sort of initial data $\left[K^{a}\right]$ for $(3.3)$, the validity of our conditions, i.e. the vanishing of $\mathcal{L}_{K} T_{(i)}^{\mathcal{A}}, \mathcal{L}_{K} g_{a b}$ and $\nabla_{c}\left(\mathcal{L}_{K} g_{a b}\right)$, can be immediately justified. This is a consequence of the fact that knowledge of $\left[K^{a}\right]$, along with the equation (3.3), suffices to determine derivatives of $K^{a}$ up to any order on $\Sigma$. As already mentioned above, the fields $T_{(i)}^{\mathcal{A}}$ and $g_{a b}$, along with their derivatives up to arbitrary order, can also be determined on the initial hypersurface $\Sigma$ in any regular initial value problem. Thereby the existence of a local one-parameter group of diffeomorphisms acting on the Cauchy development which leaves the matter fields and the geometry invariant can be read off the initial data for $T_{(i)}^{\mathcal{A}}$ and $g_{a b}$ in advance of having the relevant solution explicitly.

Secondly, in case of gauge fields it might seem to be too strong to require the vanishing of the Lie derivative of the field variable. It is known, for instance, that in the particular case of a Yang-Mills field the invariance of a vector potential $A_{a}$ (taking values in a Lie algebra $\mathfrak{g}$ ) under the action of a one-parameter group of diffeomorphisms associated with a vector field $K^{a}$ implies only that $\mathcal{L}_{K} A_{a}$ is equal to the gauge covariant derivative $D_{a}$ of a $\mathfrak{g}$-valued function $W$, i.e. $\mathcal{L}_{K} A_{a}=D_{a} W$. This general invariance property plays an important role whenever there are several symmetries acting simultaneously on a given spacetime. Note, however, that in the present situation - when we look for a single candidate Killing vector field determined by (3.3) and suitable initial data specifications - the function $W$ can be gauged away (see, e.g., page 19 of [5]) so that the invariance of an adapted vector potential $A_{a}$ can, in fact, without loss of generality, be expressed as $\mathcal{L}_{K} A_{a}=0$.

Proof: To start off consider a vector field $K^{a}$ satisfying (3.3) but which is kept otherwise to be arbitrary. Then, by taking the covariant derivative of (3.3), commuting derivatives and applying the contracted Bianchi identity, it can be shown that $\mathcal{L}_{K} g_{a b}$ satisfies the equation

$$
\nabla^{e} \nabla_{e}\left(\mathcal{L}_{K} g_{a b}\right)=-2 \mathcal{L}_{K} R_{a b}+2 R_{a b}^{e}{ }^{f}\left(\mathcal{L}_{K} g_{e f}\right)+2 R_{(a}^{e}\left(\mathcal{L}_{K} g_{b) e}\right) .
$$

Moreover, by taking the Lie derivative of (2.3) we get

$$
\mathcal{L}_{K} R_{a b}=\sum_{(i)}\left(\frac{\partial R_{a b}}{\partial T_{(i)}^{\mathcal{A}}}\right) \mathcal{L}_{K} T_{(i)}^{\mathcal{A}}+\left(\frac{\partial R_{a b}}{\partial g_{e f}}\right) \mathcal{L}_{K} g_{e f} .
$$

Then, in virtue of (3.4) and (3.5), $\mathcal{L}_{K} g_{a b}$ satisfies an equation of the form

$$
\nabla^{e} \nabla_{e}\left(\mathcal{L}_{K} g_{a b}\right)=P_{a b}\left(\mathcal{L}_{K} g_{c d}\right)+\sum_{(i)} Q_{(i)} a b\left(\mathcal{L}_{K} T_{(i)}^{\mathcal{A}}\right)
$$

where $P_{a b}$ and $Q_{(i)} a b$ are linear and homogeneous functions of their indicated arguments.

The next step is to show that $\mathcal{L}_{K} T_{(i)}^{\mathcal{A}}$ satisfy an equation analogous to 2.1) so that the coefficient of the principal part of the relevant equation is equal to that of (2.1). To achieve this take the Lie derivative of (2.1) with respect to the vector field $K^{a}$. This yields the relation

$$
\begin{aligned}
\sum_{(j)} K_{(i)(j)}^{\mathcal{A} e} \mathcal{B}\left[\mathcal{L}_{K}\left(\nabla_{e} T_{(j)}^{\mathcal{B}}\right)\right] & +\sum_{(j)}\left\{\nabla_{e} T_{(j)}^{\mathcal{B}}\left[\sum_{(k)}\left(\frac{\partial K_{(i)(j)}^{\mathcal{A} e} \mathcal{B}}{\partial T_{(k)}^{\mathcal{C}}}\right) \mathcal{L}_{K} T_{(k)}^{\mathcal{C}}+\left(\frac{\partial K_{(i)(j)}^{\mathcal{A}}}{\partial g_{c d}}\right) \mathcal{L}_{K} g_{c d}\right]\right\} \\
& +\sum_{(l)}\left(\frac{\partial L_{(i)}^{\mathcal{A}}}{\partial T_{(l)}^{\mathcal{C}}}\right) \mathcal{L}_{K} T_{(l)}^{\mathcal{C}}+\left(\frac{\partial L_{(i)}^{\mathcal{A}}}{\partial g_{c d}}\right) \mathcal{L}_{K} g_{c d}=0
\end{aligned}
$$

${ }^{5}$ Whenever $T^{\mathcal{A}}$ and $S^{\mathcal{B}}$ are tensor fields of type $(k, l)$ and $(m, n)$, respectively, $\left(\partial T^{\mathcal{A}} / \partial S^{\mathcal{B}}\right)$ is considered to be a tensor field of type $(k+n, l+m)$. Accordingly, the contraction $\left(\partial T^{\mathcal{A}} / \partial S^{\mathcal{B}}\right) \mathcal{L}_{K} S^{\mathcal{B}}$ is again a tensor field of type $(k, l)$. 
To put (3.7) into the form of (2.1) the commutation relation of the operators $\mathcal{L}_{K}$ and $\nabla_{a}$ is needed to be used which reads as

$$
\mathcal{L}_{K}\left(\nabla_{c} T_{(i)}^{\mathcal{A}}\right)=\nabla_{c}\left(\mathcal{L}_{K} T_{(i)}^{\mathcal{A}}\right)+\sum_{s=1}^{k_{i}}\left(T_{(i)}^{\mathcal{A}}\right){ }_{\left[a_{s}\right]}^{e}\left[\nabla \mathcal{L}_{K} g\right]_{e^{a}}^{a_{s}}-\sum_{t=1}^{l_{i}}\left(T_{(i)}^{\mathcal{A}}\right){ }^{\left[b_{t}\right]}\left[\nabla \mathcal{L}_{K} g\right]_{b_{t}}{ }^{e},
$$

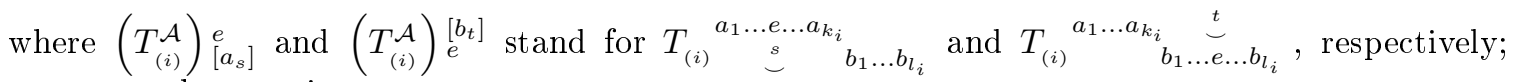
moreover, the notation

$$
\left[\nabla \mathcal{L}_{K} g\right]_{a b}{ }^{c}=\frac{1}{2} g^{c f}\left\{\nabla_{a}\left(\mathcal{L}_{K} g_{f b}\right)+\nabla_{b}\left(\mathcal{L}_{K} g_{a f}\right)-\nabla_{f}\left(\mathcal{L}_{K} g_{a b}\right)\right\}
$$

has been applied. Then, in virtue of (3.7) and (3.8), the desired equation reads as

$$
\sum_{(j)} K_{(i)(j)}^{\mathcal{A} e} \mathcal{B} \nabla_{e}\left(\mathcal{L}_{K} T_{(j)}^{\mathcal{B}}\right)+\widehat{L}_{(i)}^{\mathcal{A}}=0
$$

where the coefficients of the terms $\nabla_{e}\left(\mathcal{L}_{K} T_{(j)}^{\mathcal{B}}\right)$ are exactly that of $\nabla_{e} T_{(j)}^{\mathcal{B}}$ in $(2.1)$, and, the $\widehat{L}_{(i)}^{\mathcal{A}}$ stand for a sum of terms which are linear and homogeneous expressions of either of the variables $\mathcal{L}_{K} T_{(i)}^{\mathcal{A}}$, $\mathcal{L}_{K} g_{a b}$ or $\nabla_{c}\left(\mathcal{L}_{K} g_{a b}\right)$, respectively.

To complete our argument notice first that, in virtue of the coincidence of the principal parts of (3.10) and (2.1), along with condition 2.2, the same hyperbolization procedure that was supposed to exist in case of (2.1) can also be used to deduce from (3.10) a symmetric hyperbolic system for the variables $\mathcal{L}_{K} T_{(i)}^{\mathcal{A}}$.6 Similarly, the linear wave equation (3.6) can be put into the form of a symmetric hyperbolic system for the variables $\mathcal{L}_{K} g_{a b}$ and $\nabla_{c} \mathcal{L}_{K} g_{a b}$. Since the coupling of these two systems happens only through terms of zero order the coupled system immediately gives rise to a symmetric hyperbolic system for the fields $\mathcal{L}_{K} T_{(i)}^{\mathcal{A}}, \mathcal{L}_{K} g_{a b}$ and $\nabla_{c}\left(\mathcal{L}_{K} g_{a b}\right)$. In addition the 'source term' of the resulting system is a sum of expressions which are linear and homogeneous in either of these variables. This property guarantees that the combined equation necessarily have the identically zero solution for vanishing initial data. Since the symmetric first order system derived from (3.6) and (3.10) is linear and homogeneous in the basic variables the constraints, that there might be yielded by the relevant hyperbolic reduction of these equations, are immediately satisfied by the identically zero solutions of the coupled evolution equations. Hence whenever initial data $\left[K^{a}\right]$ can be chosen on an initial hypersurface $\Sigma$ so that $\mathcal{L}_{K} T_{(i)}^{\mathcal{A}}, \mathcal{L}_{K} g_{a b}$ and $\nabla_{c}\left(\mathcal{L}_{K} g_{a b}\right)$ are zero on $\Sigma$ then each of the fields $\mathcal{L}_{K} T_{(i)}^{\mathcal{A}}$ and $\mathcal{L}_{K} g_{a b}$ will identically vanish throughout the domain where the associated unique solution of (3.3) does exist. To see, finally, that this domain has to coincide with $D[\Sigma]$ note that, since (3.3) is a linear homogeneous wave equation any solution of it can be shown - by making use of the 'patching together local solutions' techniques described e.g. on page 266 of $[16]$ - to extend over the entire domain of dependence $D[\Sigma]$ of $\Sigma$.

\section{Concluding remarks}

In virtue of the above result one may conclude that for any gravity-matter systems satisfying conditions 2.1 and 2.2 Killing symmetries of initial data specifications $\left(\Sigma,\left[T_{(i)}^{\mathcal{A}}\right],\left[g_{a b}\right]\right)$ are preserved by the

\footnotetext{
${ }^{6}$ Recall that a hyperbolic reduction of an equation of the type (2.1), whenever it can be made, is achieved by a regular algebraic procedure (see e.g. page 9 of |11|) which depends on the principal part of the equation exclusively. Thereby, however different the source terms $L_{(i)}^{\mathcal{A}}$ and $\widehat{L}_{(i)}^{\mathcal{A}}$ might be, their difference has no influence on the hyperbolization of equation (3.10).
} 
"time" evolution. This section is to consider situations where either of the conditions of the above theorem fails to be satisfied.

Let us start by considering the evolution of certain fields on a fixed non-dynamical spacetime $\left(M, g_{a b}\right)$. Suppose that $K^{a}$ is a vector field on $M$ satisfying (3.3) but the Lie derivative of the metric with respect to $K^{a}$ should not necessarily vanish. It is straightforward to see that whenever $\mathcal{L}_{K} g_{a b}$ does not vanish the relevant form of (3.6) gives rise to a constraint equation which has to be satisfied by the Lie derivatives of fields $T_{(i)}^{\mathcal{A}}$. Furthermore, (3.10) determining the evolution of $\mathcal{L}_{K} T_{(i)}^{\mathcal{A}}$ will have non-trivial source terms in consequence of the presence of the non-vanishing fields $\mathcal{L}_{K} g_{c d}$ and $\nabla_{e} \mathcal{L}_{K} g_{c d}$. This implies then that despite of the fact that the fields $T_{(i)}^{\mathcal{A}}$ might be arranged to possess certain type of symmetries on the initial hypersurface the evolution of the system will suppress this property unless $K^{a}$ is a Killing vector field of the fixed background spacetime.

The situation is very similar, although the equations simplify considerably, whenever $K^{a}$ is chosen to be a Killing vector field for the fixed background geometry but the matter fields are kept to be general. Then the non-invariance of the matter fields - represented by the Lie derivatives $\mathcal{L}_{K} T_{(i)}^{\mathcal{A}}-$ 'evolve' according to a linear homogeneous equation of the form

$$
\sum_{(j)} K_{(i)(j)}^{\mathcal{A} e} \mathcal{B} \nabla_{e}\left(\mathcal{L}_{K} T_{(j)}^{\mathcal{B}}\right)+\sum_{(k)} L_{(k)}^{\mathcal{A}} \mathcal{B}\left(\mathcal{L}_{K} T_{(k)}^{\mathcal{B}}\right)=0
$$

where $L_{(k)}^{\mathcal{A}} \mathcal{B}$ are some smooth fields, moreover, the Lie derivatives $\mathcal{L}_{K} T_{(i)}^{\mathcal{A}}$ have also to satisfy the additional constraint

$$
\sum_{(i)}\left(\frac{\partial R_{a b}}{\partial T_{(i)}^{\mathcal{A}}}\right) \mathcal{L}_{K} T_{(i)}^{\mathcal{A}}=0 .
$$

An analogous argument applies whenever the geometry is chosen to be dynamical and initial data is arranged for (3.3) so that $\mathcal{L}_{K} g_{c d}$ and $\nabla_{e} \mathcal{L}_{K} g_{c d}$ vanish identically on $\Sigma$, however, either of the Lie derivatives $\mathcal{L}_{K} T_{(i)}^{\mathcal{A}}$ is not zero there. Then the unique solution $K^{a}$ of (3.3) will not give rise to a Killing vector field of the coupled gravity-matter system and, in general, neither of the fields $\mathcal{L}_{K} T_{(i)}^{\mathcal{A}}, \mathcal{L}_{K} g_{c d}$ and $\nabla_{e} \mathcal{L}_{K} g_{c d}$ will vanish on succeeding Cauchy surfaces.

Consider now a situation whenever condition 2.1 is not satisfied, i.e. suppose that either of the fields $K_{(i)}^{\mathcal{A} e} \mathcal{B}^{\mathcal{A}}$ used in (2.1) or the expression appearing on the r.h.s. of (2.3) depends explicitly on the points of the spacetime. This can occur, for instance, whenever there is an additional field present not taking part in the evolution. It is straightforward to see that then the Lie derivative of either of the fields $K_{(i)}^{\mathcal{A} e}{ }_{\mathcal{B}}, L^{\mathcal{A}}$ or $R_{a b}$ will not be a homogeneous expression of the Lie derivatives of the dynamical fields exclusively. Instead, terms containing the Lie derivative of the non-dynamical field appear in equations (3.6) and (3.10). Hence, unless this additional non-dynamical field is itself invariant under the action of the one-parameter families of diffeomorphisms associated with our to be Killing field $K^{a}$, these terms behave as source terms yielding the loss of any possible Killing symmetry of an initial data specification.

It is important to emphasize that the presented results also provide means to justify or dispel certain assumptions (usually made implicitly) which are applied e.g. in searching for exact or numerical solutions of the type of gravity-matter systems investigated here. For instance, if a system does not satisfy condition 2.1 it straightforwardly follows from the above considerations that the application of a dimensional reduction, based on the assumption that the spacetime admits a global symmetry, might not be completely consistent with the system under investigation. Clearly, it may happen that a gravity-matter system of this type has no solution which would possess a (local) one-parameter group of isometry actions which also would leave the matter fields to be invariant. 
Let us finally mention that on a similar base the results presented in this paper might be useful in testing the trustworthiness of a code in numerical simulation of gravity-matter systems. In particular, in case of theories satisfying conditions 2.1 and 2.2 one can be sure that something has to be wrong with a code if it does not preserve (at least approximately) symmetries of initial data specifications.

\section{References}

[1] F. Cagnac: Problème de Cauchy sur un conoüde caractéristique pour des équations quasilinéaires, Annali Mat. pura appl. 129, 12-41 (1980)

[2] P.T. Chruściel: On uniqueness in the large of solutions of Einstein's equations (Strong Cosmic Censorship). (Proc. of CMA Vol. 27. Australian National University 1991)

[3] B. Coll: On the evolution equations for Killing fields J. Math. Phys. 18, 1918-1922 (1977)

[4] R. Courant and D. Hilbert: Methods of mathematical physics Vol. II., New York, Interscience Publishers (1962)

[5] P. Forgács and N.S. Manton: Space-time symmetries in gauge theories, Commun. Math. Phys. 72, 15-35 (1980)

[6] F.G. Friedlander: The wave equation on curved space-time, Cambridge Univ. Press. (1975)

[7] H. Friedrich: On the hyperbolicity of Einstein's equations and other gauge field equations, Commun. Math. Phys. 100, 525-543 (1985)

[8] H. Friedrich: Hyperbolic reductions for Einstein's equations, Class. Quant. Grav. 13, 1451-1469 (1996)

[9] H. Friedrich, I. Rácz and R.M. Wald: On rigidity of spacetimes with stationary event- or compact Cauchy horizons, Commun. Math. Phys. 204 691-707 (1999)

[10] H. Friedrich and A. Rendall: The Cauchy problem for the Einstein equations, (Springer, Lecture Notes in Physics 540 127-224, 2000)

[11] R. Geroch: Partial differential equations of physics, in General Relativity (Proc. 46th Scottish Universities Summer School in Physics) ed. G.S. Hall and J.R. Pulham (SUSSP Publications, Edinburgh; IOP London, 1996)

[12] T. Jacobson and G. Kang: Increase of black hole entropy in higher curvature gravity, Phys. Rev. D. 52, 3518-3528 (1995)

[13] J. Kánnár: On Killing vectors in initial value problems for asymptotically flat space-times, Class. Quant. Grav. 17, 4981-4990 (2000)

[14] V. Moncrief: Spacetime symmetries and linearization stability of the Einstein equations I., J. Math. Phys. 16, 493-498 (1975)

[15] V. Moncrief: Spacetime symmetries and linearization stability of the Einstein equations II., J. Math. Phys. 17, 1893-1902 (1976)

[16] H. Müller zum Hagen and H.-J. Seifert: On characteristic initial-value and mixed problems, Gen. Rel. Grav. 8, 259-301 (1977)

[17] H. Müller zum Hagen: Characteristic initial value problem for hyperbolic systems of second order differential systems, Ann. Inst. Henri Poincaré 53, 159 - 216 (1990) 
[18] I. Rácz: On further generalization of the rigidity theorem for spacetimes with a stationary event horizon or a compact Cauchy horizon, Class. Quant. Grav. 17, 153-178 (2000)

[19] I. Rácz: On the existence of Killing vector fields, Class. Quant. Grav. 16 1695-1703 (1999)

[20] A.D. Rendall: Reduction of the characteristic initial value problem to the Cauchy problem and its applications to the Einstein equations, Proc. R. Soc. Lond. A 427, 221-239 (1990)

[21] R.M. Wald: General relativity, University of Chicago Press, Chicago (1984) 